\title{
A Modified IEEE 802.15.4 Superframe Structure for Guaranteed Emergency Handling in Wireless Body Area Network
}

\author{
Jay Shree Ranjit, Seokjoo Shin \\ Dept. of Computer Engineering, Chosun University \\ Gwangju (S. Korea) \\ E-mail: sjshin@ chosun.ac.kr $\dagger$ \\ $\uparrow$ Corresponding Author
}

Received: March 13, 2013

Accepted: June 18, 2013

Published: June 30, 2013

DOI: $10.5296 / n p a . v 5 i 2.3375$

URL: http://dx.doi.org/10.5296/npa.v5i2.3375

\begin{abstract}
Wireless Body Area Network (WBAN) is a special purpose personal area network with health-care and other value-added applications. Recently, patient monitoring applications using WBAN have received significant attention. Patient monitoring applications are intended to report any sign of emergency in the monitored patient to the corresponding health personal so that necessary actions can be taken before it is too late. Therefore, the underlying protocol suite in WBAN should ensure that the emergency events will be timely reported. However, WBAN based on IEEE 802.15.4 might not be able to do so. Hence, in this paper, we devise the emergency handling capability in IEEE 802.15.4 MAC. We propose a modified superframe structure for supporting immediate emergency reporting and reliable data transmission. A fraction of inactive period is used opportunistically for three new periods in such a way that temporary switching between the new periods and inactive period occurs only for handling emergency events. We also suggest two different random channel access mechanisms for reporting emergencies. Through the numerical results, we show that the proposed scheme achieves improved latency and throughput in the WBAN environment with compare to IEEE 802.15.4.
\end{abstract}

Keywords: Emergency reporting and handling, IEEE 802.15.4, MAC protocol, WBAN, Superframe, Opportunistic use. 


\section{Introduction}

Wireless personal area network (WPAN) is a short-range communication network. It generally covers the area around personal workspace [1]. Wireless Body Area Network (WBAN) is a special purpose WPAN with diverse health-care and other value-added applications. Among its several applications, patient monitoring has been considered as the most prominent one [2]. For patient monitoring applications, varieties of miniature sensing and communicating devices are deployed inside, on the surface or around the human body which report the sensed events to the center where the corresponding health personals have their access.

Patient monitoring applications, in general, report two types of events: regular observatory events and occasional emergency events. The regular observations may include, but not limited to, blood pressure, body temperature and oxygen saturation level in the blood [3], while the occasional emergency events could be rapid fluctuation in ECG signals, reduced blood level in brain etc., which if not addressed urgently result in life-threatening consequences or the permanent impairment of organ(s).

Thus, it is necessary to design and engineer the overall protocol suite of WBAN in such a way that it meets all the requirements with special focus to report sensed life-threatening emergency events within the specified target delay margin (for example, $125 \mathrm{~ms}$ for critical medical data [4]). Different enhancements can be introduced in any of the protocol layer, from the transport layer to the physical layer, wherever it is deemed necessary. In this paper, we confine ourselves to the enhancements applicable to the Medium Access Control (MAC) sub-layer of the data link layer.

Recently, IEEE 802.15.6 [5] standard which defines the PHY and MAC layer specifications for WBAN has been released. But in this paper, we consider the IEEE 802.15.4[6] MAC protocol for WBAN as it has been shown in [7] that for very small payloads IEEE 802.15.4 performs better in terms of average packet loss ratio and average delay than IEEE 802.15.6 due to the different CSMA strategy [5][6]. As the emergency data are also the small payload sized data [8], IEEE 802.15.4 has advantages in terms of network performances. But still as to be explained with an example in Section 2, IEEE 802.15.4 MAC protocol cannot meet the target delay requirement for reporting the emergency events in several instances.

Therefore, in this paper, we propose a modified IEEE 802.15.4 MAC frame structure that guarantees the short emergency packet handling. The inactive period of the conventional IEEE 802.15.4 MAC superframe format is modified with three new fields at the beginning solely for emergency reporting and handling purpose. Additionally, two random channel access mechanisms for reporting emergency events are suggested to resolve collisions among emergency packet transmissions. The first approach resolves the conflict in channel access among the emergent users in the code-domain utilizing CDMA codes while the second approach resolves the conflict in the time-domain using backoff window. We derive the performance bounds of the proposed scheme in terms of Throughput Upper Bound (TUB) and Delay Lower Bound (DLB) and compare to those of the conventional scheme. 
The rest of the paper is organized as follows. Some of the related works are presented and briefly discussed in Section 2. Brief overview of IEEE 802.15.4 MAC protocol is given in Section 3. The proposed scheme is explained in Section 4. Here, we discuss the two different approaches too. Analytical expressions to calculate performance limits of the proposed scheme are derived in Section 6. Numerical results are presented and discussed in Section 5. Final Section concludes the paper.

\section{Related Works}

Over a past decade, a number of MAC protocols have been researched and proposed for WBAN based on IEEE 802.15.4 standard. Amongst these protocols, many are focused to resolve energy efficiency issue while others are focused on QoS provisions. And within the QoS provision, there are a quite few protocols which are focused on emergency handling issue. So, emergency handling is the main concern here. IEEE 802.15.4 does not have any inherent emergency handling capability. So, firstly priority can be assigned to different services. Kim et al. has proposed priority based service differentiation scheme for IEEE 802.15.4 sensor networks [9]. They have suggested two different ways for the service differentiation scheme viz. by contention window (CW) size and by backoff exponent (BE) [9]. In the scheme, the nodes will have different $\mathrm{CW}$ value and $\mathrm{BE}$ values according to their priority class. The higher priority class has lower $\mathrm{CW}$ value and $\mathrm{BE}$ values. The scheme is applied during the contention access period so that priority based data transmission along with the priority based GTS allocation can easily be carried out. Hence, the scheme is quite useful but not quite sufficient to handle the emergency events in the WBAN. Because, as to be discussed in Section 3.2, the delay for reporting the emergency events is higher when the emergency happens during contention free period. Some MAC protocols from [10] to [14] are briefly discussed which have the provision for emergency handling in WBAN.

Kwak et al. has proposed traffic adaptive MAC for handling emergency and on-demand traffics that maintains a table to store the traffic pattern of the nodes [10]. It also consists of configurable contention access period (CCAP) but rest of the superframe parts resembles the conventional IEEE 802.15.4 MAC. So this MAC is bound to incur undesired delay on reporting emergency as described in Section 3.2.

Lee et al. has proposed enhanced MAC protocol of IEEE 802.15.4 [11] for WBAN with enhanced superframe structure containing emergency slot (ES) for emergency handling. ES is a quite short period where data transmission is described by success or fail. The protocol contains a long CFP and inactive period follows the CFP. So emergency occurred in CFP incurs an unnecessary delay due to inactive period.

Zhang et al. [12] has differentiated the traffics into two classes: periodic and bursty, and proposed diversified CFPs for these two classes are allocated based upon the traffic arrivals in the previous superframe. CAP is also divided into two control channels: Access Channel1 (AC1) and Access Channel2 (AC2). But the protocol is bound to suffer a long delay due to the long CFPs. Since traffics of class 1 are not allowed the CFP of class 2 and vice-versa, delay 
bound is higher when different traffics occur at different CFPs.

Khaled et al. [13] has classified traffic based on critical and non-critical issues. However, their work mainly concentrated on determining the number of retransmissions based on traffic criticality and avoided the other QoS issues.

Otgonchimeg et al. [14] has proposed emergency handling MAC protocol for human body communication using emergency GTS (EGTS) in CFP. Emergency events are treated as regular events and the number of EGTS required to handle possible emergencies are calculated. The channel access mechanism in EGTS is slotted ALOHA. Problem may arise in the protocol when the multiple emergencies occur. Also in real life scenario, the emergency events are the unpredictable occasional events and allocating resources solely for those events decreases the bandwidth utilization.

Apart from the state-of-the-art emergency handling schemes in WBAN, we introduce an opportunistic emergency handling MAC scheme that strengthens the IEEE 802.15.4 MAC by reducing the emergency reporting delay in WBAN thereby providing the reliable data transmission method for emergency events.

\section{IEEE 802.15.4 MAC Protocol}

\subsection{Overview}

IEEE 802.15.4 standard defines physical layer and MAC sublayer specifications for low-rate WPAN (LR-WPAN). It specifies two operational modes: non-beacon mode and beacon-enabled mode. Contention based unslotted Carrier Sense Multiple Access/Collision Avoidance (CSMA/CA) is used in non-beacon mode, whereas hybrid access mechanism (consisting of contention-based slotted CSMA/CA and contention-free time division multiple access) is used in beacon-enabled mode. The contention-free access mechanism in beacon enabled mode is specified to allocate conflict-free Guaranteed Time Slots (GTSs) for transmitting time-critical data. But there is no such GTS mechanism in the non-beacon mode. So we limit our research on the beacon enabled mode as GTS mechanism supports reliable transmission of emergency data.

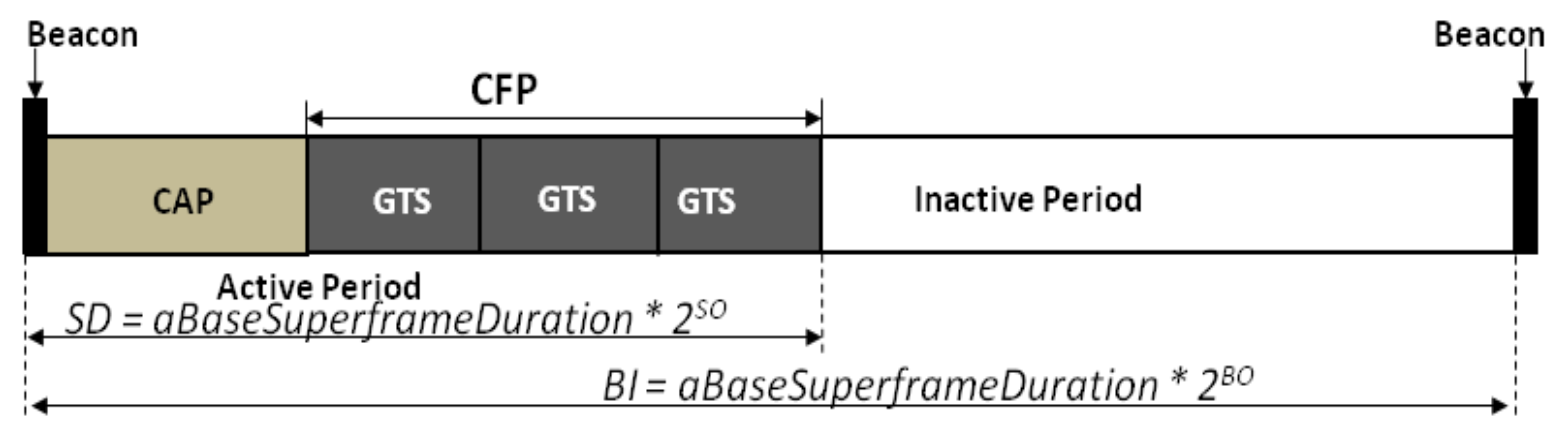

Figure 1. IEEE 802.15.4 superframe structure.

Fig.1 shows a typical IEEE 802.15.4 beacon-enabled superframe structure [6]. Every 
superframe starts with a beacon issued by the coordinator which contains information related to superframe specifications (for example Beacon Order (BO), Superframe Order (SO), duration of other fields that follows a beacon, GTS allocation schedule etc.). The duration between two consecutive beacons is called Beacon Interval (BI) which is expressed as

$$
B I=\text { aBaseSuperframeDuration } * 2^{B O}
$$

As per the standard, aBaseSuperframeDuration is 960 symbols $^{1}$ and $0 \leq \mathrm{BO} \leq 14$. A fraction of time within BI (active period) is used for accessing the medium and transmitting the data (also known as Superframe Duration (SD)) while the rest fraction is left unutilized (inactive period). These durations can be expressed as

$$
S D=\text { aBaseSuperframeDuration } * 2^{\text {SO }}
$$

and

$$
\text { InactivePeriod }=B I-S D
$$

where $0 \leq \mathrm{SO} \leq \mathrm{BO} \leq 14$. The SD consists of a mandatory Contention Access Period (CAP) and an optional Contention Free Period (CFP). CFP is activated once the resource request from a node to the PAN coordinator is received and approved.

\subsection{Inefficiency in Handling Emergency Events}

First and foremost, there is no traffic differentiation mechanism in IEEE 802.15.4. All data are treated as same so there is no way that critical or medical data gets higher priority for channel access.

Secondly, there is no priority based mechanism for GTSs allocation. Instead GTSs are served in first-in first-out (FIFO) manner. So, it is not guaranteed that emergency nodes always get GTS for guaranteed data transmission. Also, the GTS request in a frame is addressed only by the beacon in the subsequent frame, only if GTS is available. So, an undesired delay is also associated for GTS transmission.

Similarly, there is neither privilege for emergency events reporting nor any emergency handling mechanism at all. Rather, undesired delay is incurred for emergency events reporting. The delay is more severe when the emergency events occur during CFP. For example, if an emergency event occurs at the beginning of CFP, it will be reported only in the subsequent CAP (in the next frame) or else the emergency event demands GTS then it first has to first contend for GTS in the subsequent CAP and will be scheduled with GTS in the next's next frame if only the GTS is available. As an trivial example, if we consider the CFP of a superframe with BO 4 and SO 3 (length of superframe as such is $256.76 \mathrm{~ms}$ ) consists of the 7 GTS with each GTS expanding to a slot, then as such the emergency events occurring at the beginning of CFP will be considered for access at least only after $176.76 \mathrm{~ms}$ in the subsequent CAP (considering the summation of CFP and inactive period only) which is

\footnotetext{
11 symbol duration is $16 \mu \mathrm{s}$.
} 
higher than the target delay limit for reporting emergency events, $125 \mathrm{~ms}$ [4].

\section{The Proposed Scheme}

In this section, we describe the modifications introduced in the conventional IEEE 802.15.4 superframe in order to efficiently report emergency events. We also suggest and describe the two different channel access mechanisms that can be opted in emergency reporting period.

\subsection{Overview}

In the conventional IEEE 802.15.4, the nodes and the coordinator go to sleep mode in the inactive period (if exists) after the CFP ends. In our proposed scheme we use this inactive period opportunistically. The modified frame format is similar to the original format except a fraction of inactive period is utilized to introduce three new fields: i). Emergency Reporting Period (ERP), ii). Emergency Beacon (EB) and iii). Emergency Transmission Period (ETP). ERP is a mandatory period while EB and ETP are optional ones. Only the emergency Body Nodes (BNs) are allowed to report and transmit emergency data in these periods while the rest of the BNs go to sleep mode as in inactive period of conventional IEEE 802.15.4. Fig. 2 shows the modified frame format.

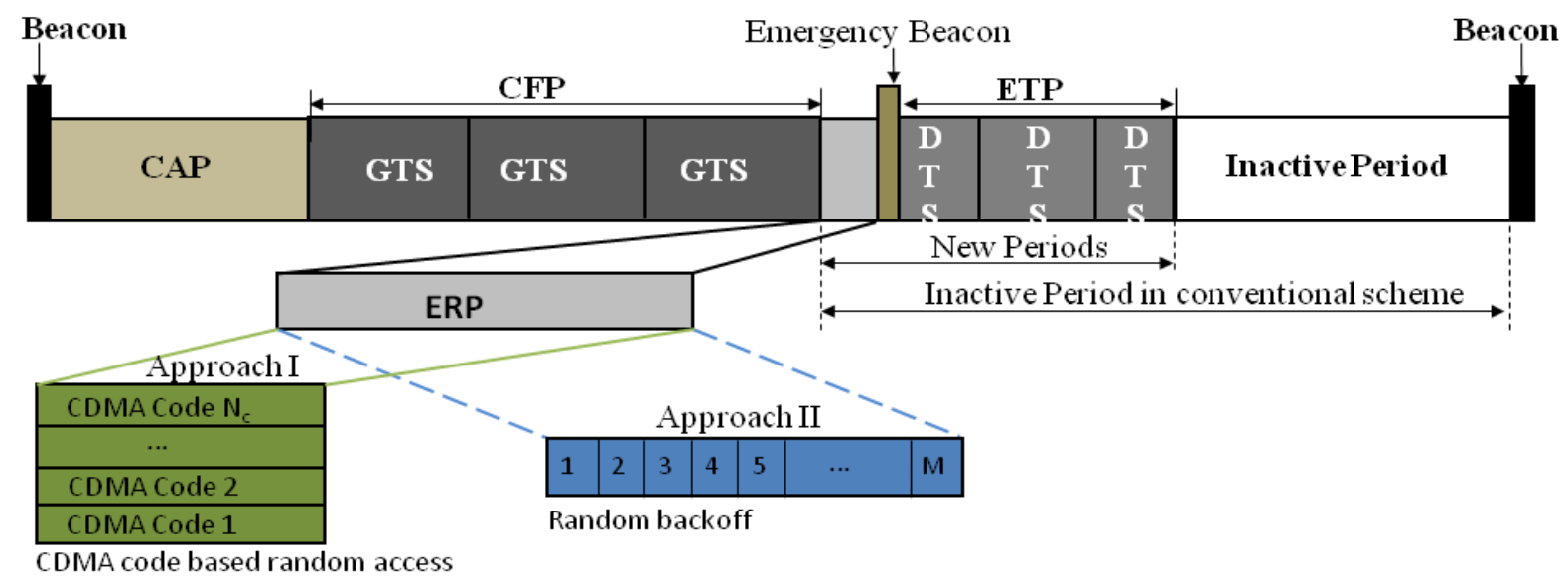

Figure 2. Modified superframe structure of the proposed scheme.

ERP starts just after the CFP ends. In order to notify this ERP information to all BNs, the conventional beacon is modified to incorporate the ERP start time and ERP length. Any of the random access mechanisms can be followed to access the channel during ERP but in this paper we suggest two different random access mechanisms i.e.. i). CDMA code based random access and ii). Random backoff based random access. These two mechanisms are briefly discussed in the following sub-section 2. In ERP, BNs report emergency events to the WBAN coordinator (BNC) either by transmitting the acquired CDMA codes in CDMA code based contention or the DTS allocation request in case of random backoff approaches.

EB follows the ERP. But EB is broadcasted by the BNC, only if any emergency is 
reported during ERP, otherwise EB period is used as inactive period. EB contains the transmission schedules (i.e. allocated Dedicated Transmission Slots (DTS)) in the following ETP. All the BNs who have reported emergency in ERP should listen to EB to check whether their reporting in ERP are acknowledged by BNC and are allocated with DTS schedule.

ETP is a TDMA based access period which appears after EB. ETP is divided into a number of slots called as DTS. The length of a DTS slot is so determined to accommodate an emergency data and an ACK message. The number of such DTSs is limited to seven.

If any of the emergency BNs is not able to report the emergency during ERP or not scheduled with DTS in the EB message or doesn't receive ACK for its data transmission in DTS, then it waits till the next CAP for data transmission.

\subsection{Channel Access Mechanisms in ERP}

In this subsection, we briefly describe the two random multiple access approaches used for channel access during ERP i.e. CDMA code based contention approach and random backoff based contention approach.

CDMA Code Based Contention Approach: In this approach, if a $\mathrm{BN}$ detects an emergency event during CFP, it waits until the ERP starts and then transmits a randomly selected CDMA code from a given code-set of $\mathrm{N}_{\mathrm{c}}$ codes. As such, collision may happen when more than one $\mathrm{BN}$ select and transmit the same code. This may happen frequently when number of emergency BNs exceeds the available CDMA codes. So in order to reduce such collisions, the access of random CDMA code is controlled by code access permission probability $\left(P_{c a}\right)$ which is broadcasted by $\mathrm{BNC}$ in its regular beacon. $P_{c a}$ is determined by BNC based on its observation regarding the number of emergency events $(\Delta N)$ in the previous ERP. The number of events can be roughly estimated through the multiplicity level of the received power at the ERP. Once $\Delta \mathrm{N}$ is estimated, value for $P_{c a}$ can be assigned using the following relation:

$$
P_{c a}=\left\{\begin{array}{cc}
1 & \text { if } \Delta N \leq N_{c} \\
\frac{N_{c}}{\Delta N} & \text { if } \Delta N>N_{c}
\end{array}\right.
$$

Random Backoff Based Contention Approach: In this approach, the ERP is divided into $\mathrm{M}$ number of backoff slots with each slot having duration sufficient enough to send one DTS Request Packet (DRP) from the $\mathrm{BN}$ and receive DRP ACK from BNC. If a BN detects an emergency event during CFP, it waits until the start of ERP and randomly selects a backoff value within $[0, \mathrm{M}]$. At the elapse of a backoff slot duration, the BN decreases its backoff counter by 1 and checks whether the backoff counter is zero. The process continues until the counter ultimately goes to zero. Once the backoff counter value reaches 0 , the BN sends a DTS Request Packet (DRP) and waits for ACK. 


\section{MInstitute"}

\section{Performance Metrics}

In this section, we formulate some expressions to calculate the two important performance metrics related to delay and throughput. We name those two metrics as Delay Lower Bound (DLB) and Throughput Upper Bound (TUB). It is worth mentioning that DLB and TUB are not the average delay and throughput performances, rather they are the bounding performances (i.e. maximum achievable). Such performance can be achieved only in ideal conditions when there is no packet failure in the network either due to collision or channel error. In what follows, we calculate DLB and TUB for a BN which need to report emergency events occurred during CFP interval.

\subsection{Delay Lower Bound}

For sending a packet of size $\mathrm{x}$, DLB for the conventional scheme can be calculated using the following expression:

$$
D L B_{C}(x)=T_{C F P}+T_{\text {Inactive }}+T_{\text {Beacon }}+T_{S I F S}+T_{B O}+T_{(x)}+T_{T A}+T_{A C K}+T_{I F S(x)}
$$

where $T_{C F P}$ is duration of CFP, $T_{\text {Inactive }}$ is the duration of the inactive period, $T_{\text {Beacon }}$ is the beacon duration, $T_{(x)}$ is the time taken to transmit packet of size $\mathrm{x}, \mathrm{T}_{\mathrm{TA}}$ is the turnaround time, $\mathrm{T}_{\mathrm{ACK}}$ is the time taken for ACK transmission, $\mathrm{T}_{\mathrm{IFS}(\mathrm{x})}$ is the IFS time for packet of size $\mathrm{x}$, and $\mathrm{T}_{\mathrm{BO}}$ is the duration of backoff period. Note that in IEEE 802.15.4, $\mathrm{T}_{\mathrm{IFS}(\mathrm{x})}$ is $640 \mu \mathrm{s}$ for $\mathrm{x}$ greater than 18 Bytes, otherwise it is $192 \mu \mathrm{s}$. During backoff procedure in beacon enabled IEEE 802.15.4, a node performs clear channel assessment (CCA) twice and hence backoff duration can be expressed as

$$
T_{B O}=B O_{\text {slots }} * T_{B O_{-} \text {slot }}+2 T_{C C A}
$$

where $\mathrm{BO}_{\text {slots }}$ is the average number of slots that the node deferred its transmission, $\mathrm{T}_{\mathrm{BO} \_ \text {slot }}$ is the slot $(320 \mu \mathrm{s})$ duration, and $\mathrm{T}_{\mathrm{CCA}}$ is the time required for CCA.

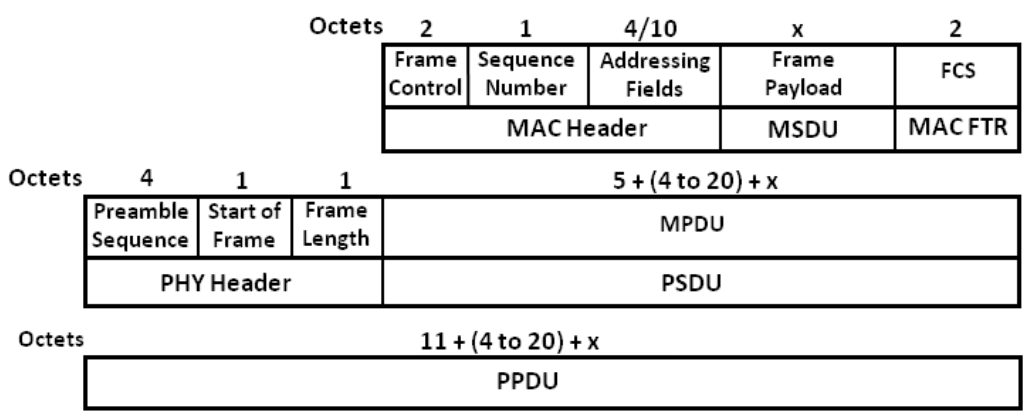

Figure 3. Frame format (IEEE 802.15.4).

Based on frame format shown in Fig. 3, we calculate $\mathrm{T}_{(\mathrm{x})}$ to be

$$
T_{(x)}=8 \cdot \frac{L_{P H Y}+L_{M A C_{-} H D R}+L_{\text {address }}+x+L_{M A C_{-} F T R}}{R_{\text {data }}}
$$


where $\mathrm{L}_{\mathrm{PHY}}$ is the length of the PHY header in bytes, $\mathrm{L}_{\mathrm{MAC} \_H D R}$ is the length of the MAC header in bytes, $\mathrm{L}_{\text {address }}$ is the length of the MAC address, $\mathrm{L}_{\text {MAC_FTR }}$ is the length of the MAC footer in bytes, and $\mathrm{R}_{\mathrm{data}}$ is the raw data rate in bits per second (bps). Finally, $\mathrm{T}_{\mathrm{ACK}}$ in (5) can be calculated as

$$
T_{A C K}=8 \cdot \frac{L_{P H Y}+L_{M A C_{-} H D R}+L_{M A C_{-} F T R}}{R_{\text {data }}}
$$

We calculate the DLB for the proposed scheme by summing the durations required for packet exchange and protocol timing specifications as in the case of the conventional scheme. DLB for the proposed scheme for reporting the emergency event using $\mathrm{x}$ Byte packet is

$$
D L B_{P}(x)=T_{C F P}+T_{E R P}+T_{S I F S}+T_{E B}+T_{S I F S}+T_{(x)}+T_{T A}+T_{A C K}+T_{I F S(x)}
$$

where $T_{E R P}$ is the duration of ERP and $T_{E B}$ is the $E B$ duration. The value of $T_{E R P}$ is different for the suggested approaches. For CDMA code based contention approach, the length of $\mathrm{T}_{\mathrm{ERP}}$ is the summation of the code propagation delay $\left(\mathrm{T}_{\mathrm{CPD}}\right)$ and the code transmission time $\left(\mathrm{T}_{\mathrm{CTT}}\right)$. But since $\mathrm{T}_{\mathrm{CPD}}$ is very negligible we do not consider it in this analysis whereas for $\mathrm{T}_{\mathrm{CTT}}$ we consider each CDMA code is of 144 bits. And for the random backoff based contention approach, the length of $\mathrm{T}_{\mathrm{ERP}}$ is dependent upon the number of random backoff slots $\left(\mathrm{T}_{\mathrm{bslot}}\right)$ and is given as $\left[\mathrm{M} \mathrm{x} \mathrm{T}_{\mathrm{bslot}}\right]$.

It is worthwhile to mention that the length of CFP is a variable one which is dependent on the number of GTSs allocated. At maximum 7 such GTSs can be allocated in a time provided that there is a sufficient resource available i.e. the total length of CFP must not violate the minimum length of CAP $\left(\mathrm{CAP}_{\min }=440\right.$ symbols $)$. Each GTS can occupy a single or more transmission slots. So, the smallest length of CFP denoted by $\mathrm{CFP}_{\min }$ is a single GTS extending to a single transmission slot, while the largest allowed CFP length is total length of superframe duration with deducting the beacon period and the $\mathrm{CAP}_{\min }$ i.e $\mathrm{CFP}_{\max }=\mathrm{SD}-$ $\mathrm{T}_{\text {Beacon }}-\mathrm{CAP}_{\min }$. Roughly, the average length of $\mathrm{CFP}\left(\mathrm{CFP}_{\mathrm{avg}}\right)$ can be taken as the average of these maximum and minimum CFP lengths. On the basis of these varying CFP lengths, the different cases of DLB calculation for proposed scheme hence are calculated for best case DLB, worst case DLB and average case DLB.

\subsection{Throughput Upper Bound}

Once the DLB for the conventional scheme and the proposed scheme are known, TUB for both the schemes can be calculated as

$$
T U B_{C}=8 \cdot \frac{x}{D L B_{C}(x)} \text { and } T U B_{P}=8 \cdot \frac{x}{D L B_{P}(x)}
$$

where, $\mathrm{TUB}_{\mathrm{C}}$ and $\mathrm{TUB}_{\mathrm{P}}$ are $\mathrm{TUB}$ for the conventional scheme and the proposed scheme, respectively. 


\section{Numerical results}

In this section, we have compared DLB and TUB of the proposed scheme and the conventional scheme using the expression derived in the previous section and considering the parameters in Table 1. It is noteworthy to mention that in our numerical analysis emergency events are considered to occur at the beginning of CFP. Besides, three different cases for DLB and TUB viz. best, worst and average cases are presented and discussed for both the proposed and conventional scheme

Table 1. Considered parameters and their values

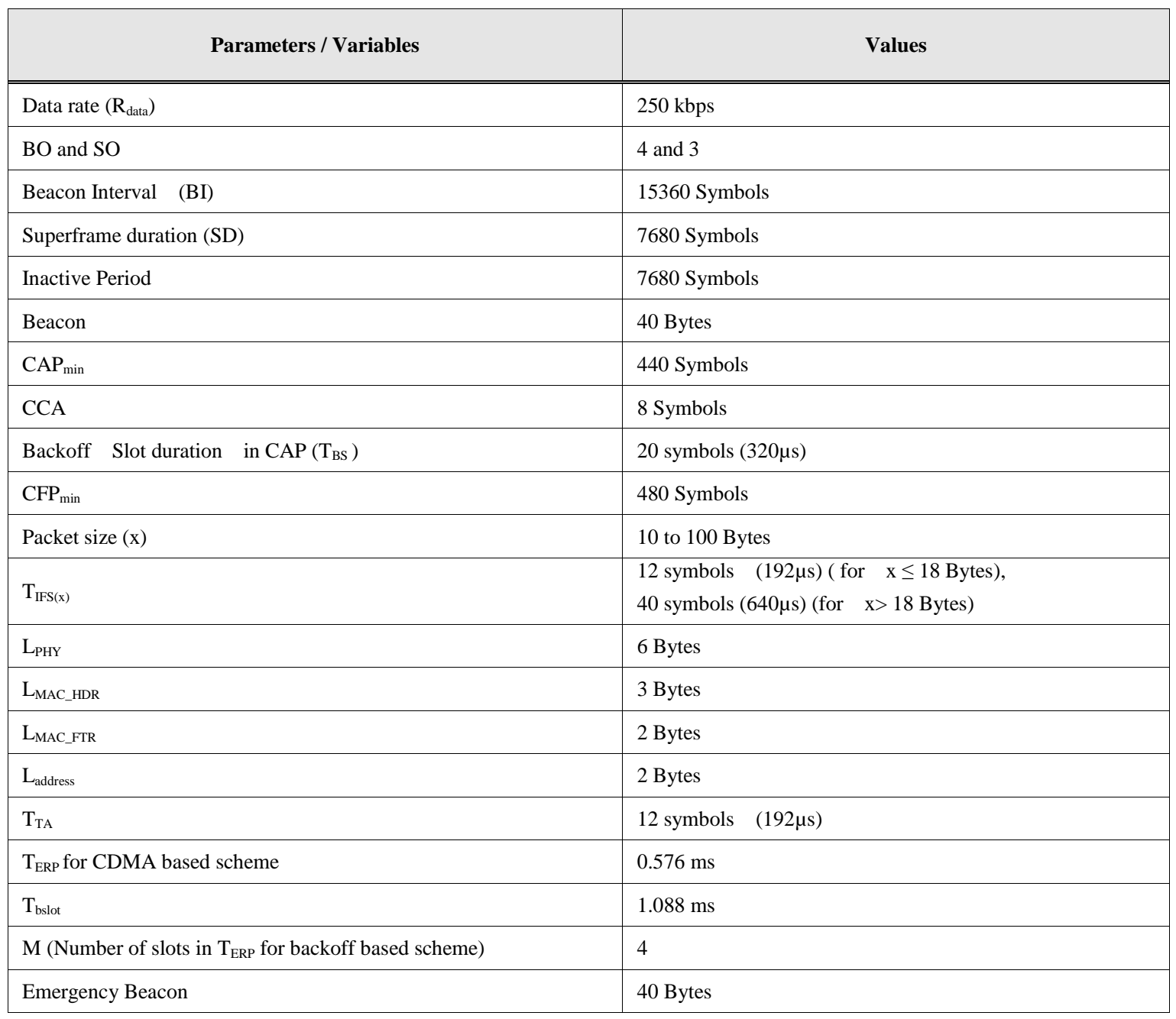




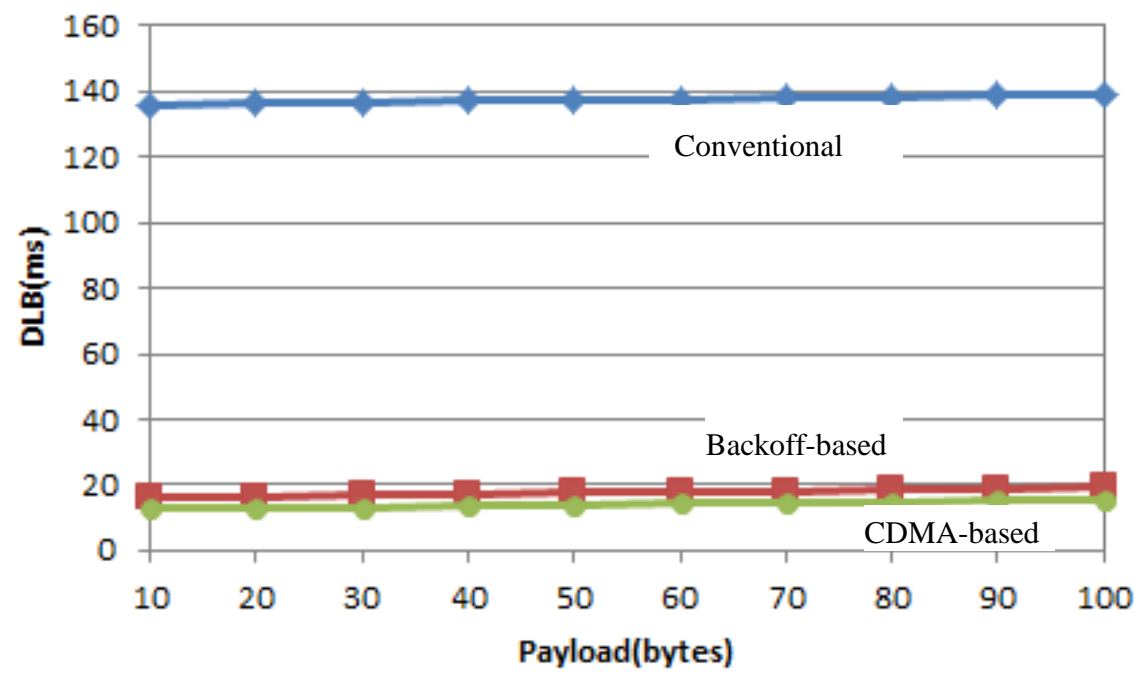

(a). DLBs of the proposed and the conventional schemes (Best Case).

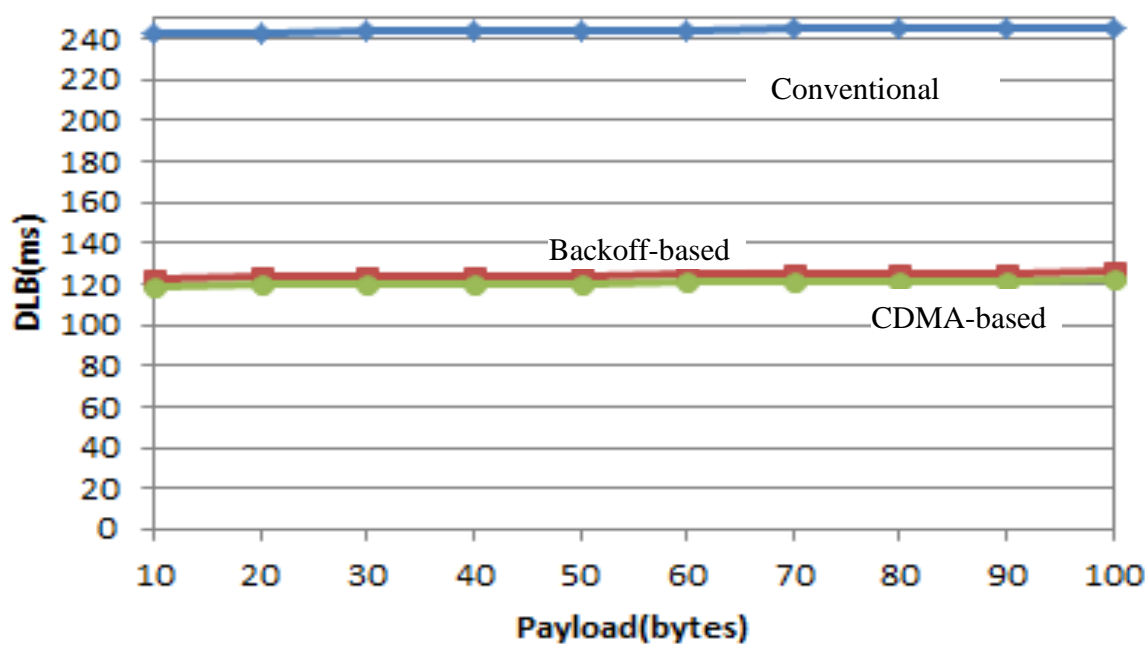

(b). DLBs of the proposed and the conventional schemes (Worst Case).

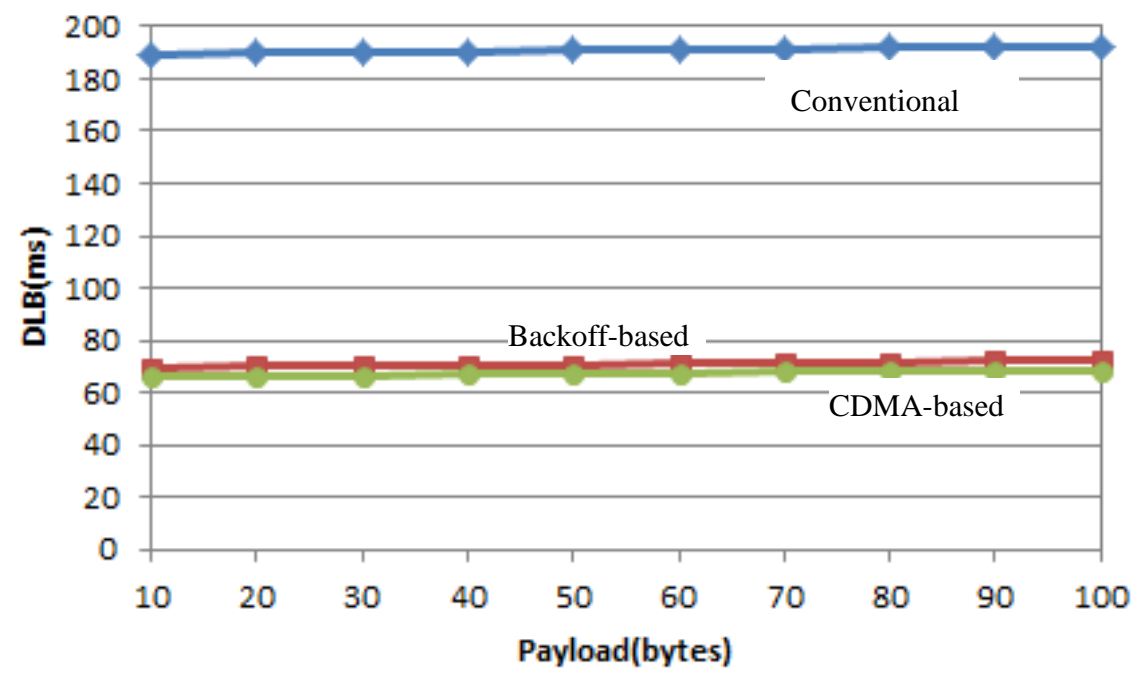

(c). DLBs of the proposed and the conventional schemes (Average Case).

Figure 4. DLBs comparison of the proposed and the conventional schemes. 
Fig. 4 shows that DLBs (for different approaches) of the proposed scheme and the conventional scheme at three different cases, best, worst and average. It is observed from the figures that, DLBs (for different approaches) of the proposed scheme are less than that of the conventional scheme, regardless of the payload size. In either of the cases, the proposed scheme has the DLB lower than the delay requirement of WBAN whereas the DLBs in conventional scheme do not meet this target. This makes proposed scheme much more favorable for WBAN applications. Among the suggested two approaches, the CDMA code based scheme yields lower DLB than the backoff based approach. For example, when payload is 60 Bytes, DLB of the CDMA code based scheme is $89 \%$ and $50 \%$ lower than that of the conventional scheme for the best and worst case scenario, while DLB of the backoff based scheme is only $87 \%$ and $49 \%$ lower. The lower DLB in the CDMA-based scheme is attributed to its requirement of relatively smaller ERP than in the backoff based scheme.

Fig. 5 shows that TUBs of the proposed scheme with suggested two approaches and the conventional scheme increase linearly with increase in the payload size. Moreover, TUBs of the proposed scheme are higher than that of the conventional scheme. As in the case of DLP comparison, the CDMA code based scheme yields higher TUB than the backoff based scheme. For example, when payload is 60 Bytes, TUB of the CDMA code based scheme and backoff based schemes are $102.47 \%$ and $96.31 \%$ higher respectively for the worst case, than that of the conventional scheme.

From the above comparisons, it is seen that the CDMA based approach is comparatively more suitable for reporting emergency events in WBAN patient monitoring applications than the backoff based approach. But when the ERP length in both approaches is same then performance of these approaches will be identical.

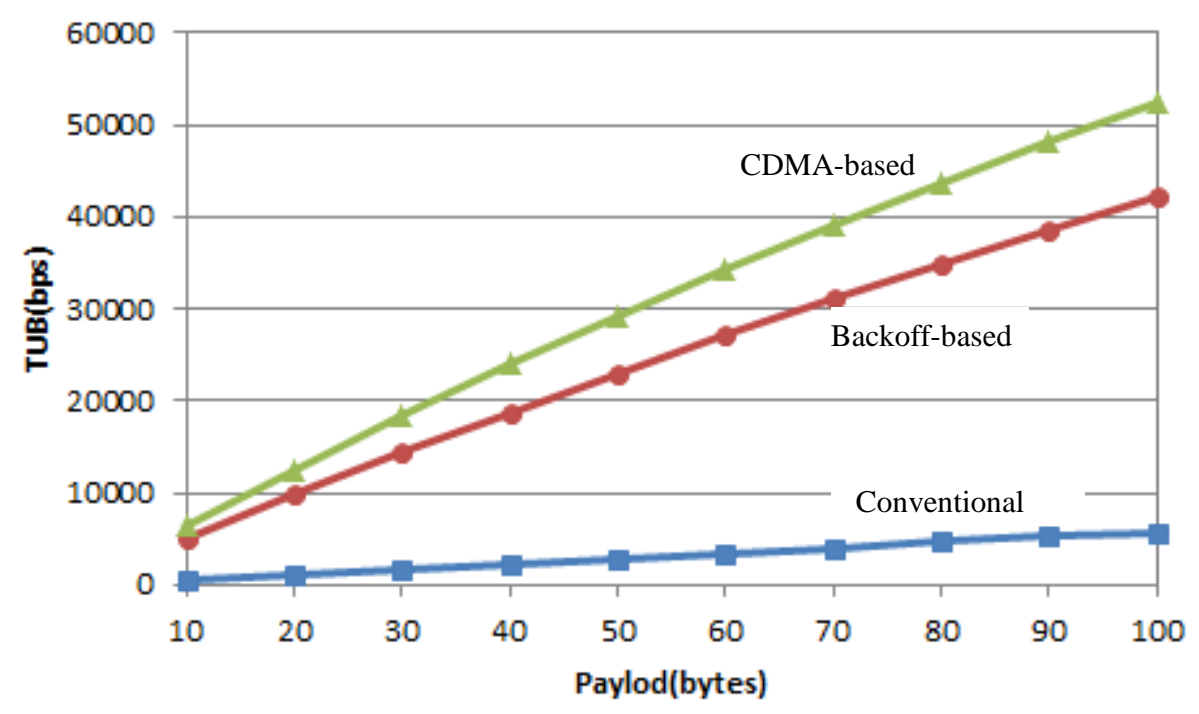

(a). TUBs of the proposed and the conventional schemes (Best Case). 


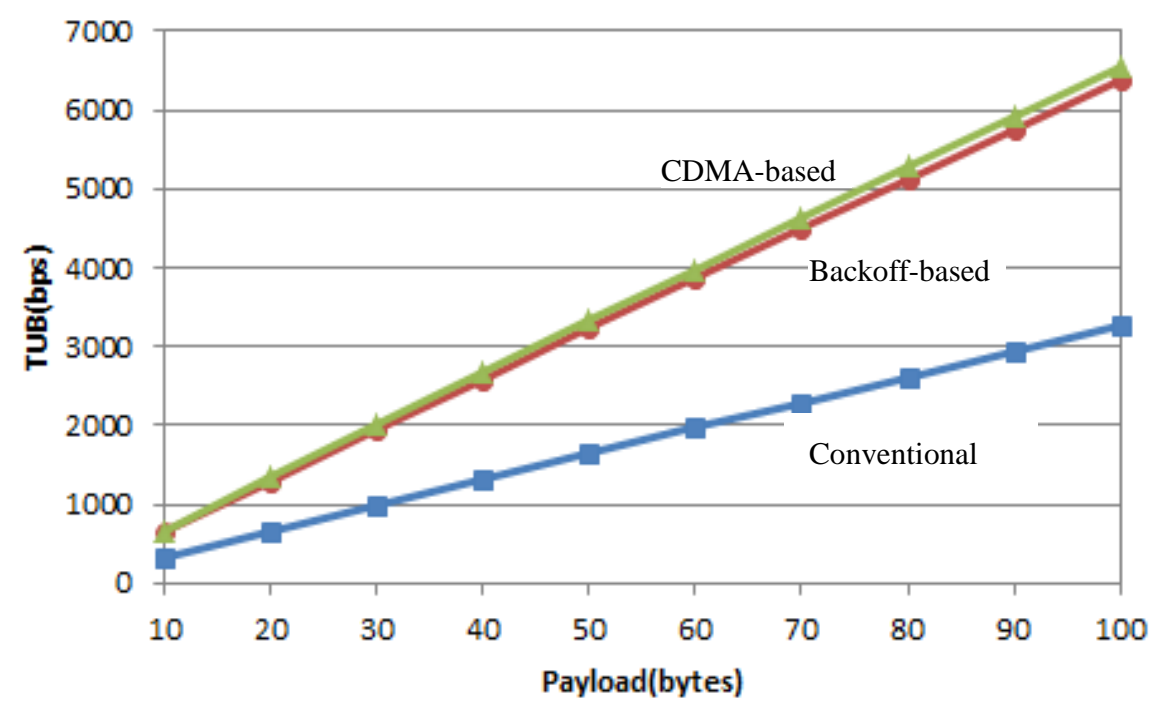

(b). TUBs of the proposed and the conventional schemes (Worst Case).

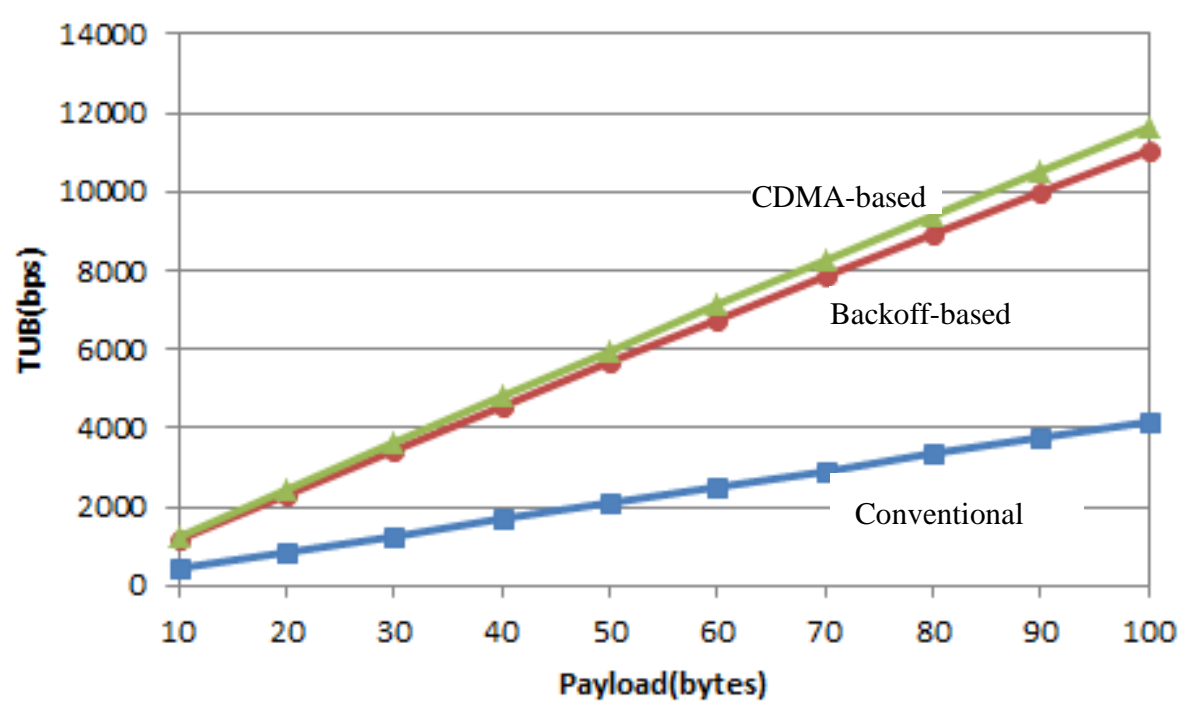

(c). TUBs of the proposed and the conventional schemes (Average Case).

Figure 5. TUBs comparison of the proposed and the conventional schemes..

\section{Conclusion and Future Works}

We proposed a modified IEEE 802.15.4 frame structure for WBAN that opportunistically uses the inactive period for handling short emergency packets. We also suggested two different channel access approaches so as to resolve the possible collisions during the emergency transmissions. The first mechanism resolves the conflict in channel access among the emergent BNs in the code-domain utilizing CDMA codes while the second approach resolves the conflict in the time-domain using backoff window. Moreover, we showed through numerical results that for the both suggested approaches, DLBs of the proposed scheme are smaller whereas TUBs are larger than those of conventional scheme. And among the two suggested approaches, the CDMA based scheme has higher TUB and lower DLB 
because of shorter ERP length. But, either of these approaches can be implemented depending upon the application needs.

As a future work, the proposed scheme will be validated by the discrete event computer simulation.

\section{Acknowledgement}

This research was supported by Basic Science Research Program through the National Research Foundation of Korea (NRF) funded by the Ministry of Education (No. 2012R1A1A2006327)

\section{References}

[1] Park W. K., Han I., Park K. R., "ZigBee based Dynamic Control Scheme for Multiple Legacy IR Controllable Digital Consumer Devices". Consumer Electronics. Vol. 53, Pp 172-177. February 2007. http://dx.doi.org/10.1109/ICCE.2007.341548

[2] Monton E., Hernandez J. F., Blasco J. M., Herve T., Micallef J., Grech I., Brincat A., Traver V., "Body Area Network for Wireless Patient monitoring". IET Communications. Vol. 2, Pp 215-222. February 2008. http://dx.doi.org/10.1049/iet-com:20070046

[3] Li H. M., Tan J. D., "An ultra-low-power medium access control protocol for body sensor network", 27th Annual International Conference of the Engineering in Medicine and Biology Society (IEEE-EMBS 2005). Sanghai, China, September 2005. http://dx.doi.org/10.1109/IEMBS.2005.1616964

[4] IEEE 802.15-08-0664-09-0006, TG6 Technical Requirements (TRD). Available at: https://mentor.ieee.org/802.15/documents?n=9\&is_group=0006

[5] IEEE Std. 802.15.6-2012, Part 15: Wireless Body Area Networks, 2012. Available at: http://standards.ieee.org/getieee802/download/802.15.6-2012.pdf

http://dx.doi.org/10.1109/IEEESTD.2012.6161600

[6] IEEE Std. 802.15.4-2006, Part 15: Wireless Medium Access Control (MAC) and Physical Layer (PHY) Specifications for Low-Rate Wireless Personal Area Networks (WPANs), 2003. Available at: http://book.itep.ru/depository/zigbee/802-15-4-2006.pdf

[7] Martelli F., Buratti C., Verdone R., "On the performance of an IEEE 802.15.6 wireless body area network", 11th European Wireless Conference-Sustainable Wireless Technologies (European Wireless). Vienna, Austria, April 27-29, 2011.

[8] Chen H., Wu., Lee J., "A WBAN-based Real-time Electroencephalogram Monitoring System: Design and Implementation". Journal of Medical Systems. Vol. 34, Issue 3. Pp 303-311, March 2009. http://dx.doi.org/10.1007/s10916-008-9242-9

[9] Kim E. J., Kim M., Youm S. K., Choi S., Kang C. H., "Priority-based Service Differentiation Scheme for IEEE 802.15.4 Sensor Networks". International Journal of Electornics and Communications. Vol. 61, Issue 2. Pp 69-81, February 2007, http://dx.doi.org/10.1016/j.aeue.2006.02.004

[10]Kwak K. S., Ullah S., "A traffic-adaptive MAC protocol for WBAN", 2010 
IEEE-GLOBECOM Workshops (GC Wkshps), Miami, Florida, December 6-10, 2010. http://dx.doi.org/10.1109/GLOCOMW.2010.5700145

[11]Lee C., Lee H. S., Choi S., "An enhanced MAC protocol of IEEE 802.15. 4 for wireless body area networks", 5th International Conference of Computer Sciences and Convergence Information Technology (ICCIT), Seoul, November 30 - December 2, 2010. http://dx.doi.org/10.1109/ICCIT.2010.5711190

[12] Zhang Y., Dolmans G., "A new priority-guaranteed MAC protocol for emerging body area networks", 5th International Conference on Wireless and Mobile Communications (ICWMC '09), Cannes, La Bocca, August 23-29, 2009. http://dx.doi.org/10.1109/ICWMC.2009.30

[13] Ali K. A., Sarker J. H., Mouftah H. T., "QoS-based MAC protocol for medical wireless body area sensor networks", IEEE Symposium on Computers and Communications (ISCC2010), Riccione, Italy, June 22-25, 2010.http://dx.doi.org/10.1109/ISCC.2010.5546523 [14] Otgonchimeg B., Kwon Y., "Emergency Handling for MAC Protocol in Human Body Communication". EURASIP Journal on Wireless Communications and Networking, Vol. 1, Pp 1-6, March 2011. http://dx.doi.org/10.1155/2011/786903

\section{Copyright Disclaimer}

Copyright reserved by the author(s).

This article is an open-access article distributed under the terms and conditions of the Creative Commons Attribution license (http://creativecommons.org/licenses/by/3.0/). 